\title{
Diseño y validación de una escala de impacto psicosocial de los desastres (SPSI-D)
}

\section{[Design and validation of a scale of psychosocial impact of disasters (SPSI-D)]}

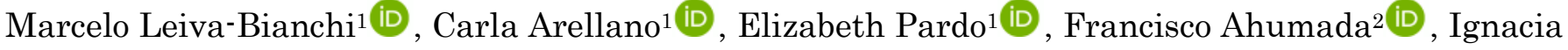 \\ Amaya $^{1}$ (D), Sylvia Antúnez ${ }^{2}$ (D) Cristian Cáceres $^{1}$ (D), Melissa Salinas ${ }^{1}$ (D), \& Carlos Serrano ${ }^{1}$ (iD) \\ 1Universidad de Talca; 2 Universidad Católica del Maule
}

\begin{abstract}
Resumen
Los desastres se caracterizan por generar un impacto en las personas y comunidades expuestas, quienes responderán de manera disruptiva o saludable. El objetivo de esta investigación es definir ese impacto psicosocial mediante el diseño y validación de la Escala de Impacto Psicosocial de los Desastres (SPSI-D). Para ello se revisó literatura centrada en el impacto de los desastres, se diseñaron ítems que fueron evaluados por jueces-expertos; y se validó la escala en una muestra de personas expuestas al terremoto y tsunami del 27 de febrero de 2010 en Chile $(n=467)$. Se utilizó el análisis factorial paralelo, confirmatorio y curvas ROC. Los resultados evidencian la validez, fiabilidad y precisión de la SPSI-D, la que quedó conformada por 51 ítems, agrupados en 6 dimensiones: exposición, protección, creencias de suerte, autoconcepto, disrupción y salud. El instrumento integra las ventajas de medir respuestas disruptivas y saludables después de un desastre. Se deja la SPSI-D a disposición de los profesionales que la requieran.
\end{abstract}

Palabras clave: impacto psicosocial, trastorno de estrés postraumático, análisis factorial paralelo, análisis factorial confirmatorio, desastres.

\begin{abstract}
Disasters have an impact on the people and communities affected, and these will respond in either a disruptive or healthy manner. The aim of the present research is to define this psychosocial impact through the design and validation of the Scale of Psychosocial Impact of Disasters (SPSI-D). A review of literature focused on the impact of disasters was conducted, items were designed and evaluated by expert judges, and the scale was validated in a sample of people affected by the earthquake and tsunami of February 27, 2010 in Chile $(n=467)$. Parallel and confirmatory factor analysis and ROC curves were used. The results show the validity, reliability and precision of the SPSI-D, which comprised 51 items grouped into 6 dimensions: exposure, protection, belief in luck, self-concept, disruption and health. The instrument brings together the advantages of measuring both disruptive and healthy responses in the wake of a disaster. SPSI-D is made available to all professionals to whom it may be of use.
\end{abstract}

Keywords: psychosocial impact, post-traumatic stress disorder, parallel factor analysis, confirmatory factor analysis, disasters.

Contacto: La comunicación sobre este artículo debe ser enviada a Marcelo Leiva-Bianchi, email: marcleiva@utalca.cl

Financiamiento: Fondo Nacional de Desarrollo Científico y Tecnológico (FONDECYT) Regular N ${ }^{\circ} 11121330$ "Construcción de un modelo predictivo del impacto psicosocial a partir de datos empíricos y simulados vinculados con el terremoto y tsunami del 27 de febrero de 2010" 


\section{INTRODUCCIÓN}

En las últimas décadas la frecuencia de los desastres ha aumentado. Entre el año 2000 y 2009 hubo tres veces más desastres que los producidos durante la década de los ochenta, lo cual se asocia principalmente a eventos climáticos, que representan casi el $80 \%$ del incremento, mientras que en los eventos geofísicos la tendencia se mantiene (Leaning \& Guha-Sapir, 2013). El aumento de la urbanización, industrialización, deforestación y degradación ambiental son factores que influyen en el cambio climático global, el cual se asocia a una mayor exposición de las personas y comunidades a desastres naturales (Guha-Sapir, Hargitt, \& Hoyois, 2004). Según el modelo de conservación de recursos de Hobfoll (1989, 2012), un desastre produce en el ambiente una demanda ecológica, disminuyendo los recursos y la capacidad tanto de las personas como de las comunidades para protegerse, recuperarse o invertir con nuevos recursos. Se debe considerar que no todas las personas están igualmente expuestas a los desastres, ni poseen los mismos recursos para afrontarlos; por ejemplo, quienes poseen menos recursos son más vulnerables a perderlos y tienen menor probabilidad de utilizar los recursos disponibles (Hobfoll, 2012). Según los criterios del Centre for Research on the Epidemiology of Disasters para que un evento se considere un desastre y se incluya en la Emergency Events Database (EM-DAT) debe cumplir con al menos uno de los siguientes cuatro criterios (Guha-Sapir, Hoyois, \& Below, 2014): 10 o más personas muertas; 100 o más personas afectadas; declaración de estado de emergencia; o una petición de ayuda internacional.

¿Qué les ocurre a las personas y a las comunidades con los desastres? Existe controversia respecto de qué es el impacto psicosocial (IPS) de eventos potencialmente traumáticos (EPT), como es el caso de los desastres. Una revisión del concepto impácto psicológico (psychological impact) e impacto psicosocial (psychosocial impact) en los títulos de revistas indexadas en ISI Web of Knowledge, indica la existencia de 638 artículos referidos a este concepto. Todos esos artículos pueden categorizarse en tres grupos: (a) impacto psicosocial de las enfermedades médicas (243 artículos); (b) impacto psicosocial de tratamientos o prácticas médicas (280 artículos); y (c) impacto psicosocial de eventos de carácter no médico (115 artículos). Es interesante notar que existen pocos estudios que definen de manera precisa el concepto. Uno de esos trabajos es el modelo del impacto psicológico y social de los atentados del 11 de septiembre de 2001 (Morgan, Wisneski, \& Skitka, 2011); allí se define operacionalmente el impacto en torno a variables positivas (cercanía con otros, donaciones y compromiso político) y negativas (hostilidad, intolerancia y prejuicios) en la población estadounidense. Otro trabajo define el modelo de IPS del cáncer a partir del continuo de variables positivas (afrontamiento, autoconcepto positivo, auto-conexión, espiritualidad y crecimiento significativo) y negativas (estrés, 
autoconcepto negativo, aislamiento social, perdida de sentido y espiritualidad; Lai, Garcia, Salsman, Rosenbloom, \& Cella, 2012). Para el caso de los desastres, diversos estudios (Belter, Dunn, \& Jeney, 1991; Davis, Grills-Taquechel, \& Ollendick, 2010; Freedy, Shaw, Jarrell, \& Masters, 1992; Jia et al., 2010; Sutker, Corrigan, Sundgaard-riise, Uddo, \& Allain, 2002; Ticehurst, Webster, Carr, \& Lewin, 1996; Viswanath et al., 2012; Weems et al., 2007) dan cuenta de variables positivas o saludables (crecimiento post-traumático, apoyo social, bienestar emocional, emociones positivas $\mathrm{y}$ afrontamiento) y negativas o disruptivas (estrés post-traumático, distrés, depresión, ansiedad, respuestas de odio, comportamiento disruptivo y psicopatología general). El continuo de variables positivas o negativas que también está presente en modelos clásicos de emociones básicas (Plutchik \& Conte, 1997; Russell, 2003; Watson $\&$ Tellegen, 1985), aparece constantemente en los estudios revisados del IPS.

Los desastres se caracterizan por impactar en distintas magnitudes a las personas y comunidades expuestas (Bonanno, 2004; Bonanno, Brewin, Kaniasty, \& Greca, 2010). De acuerdo con el modelo de exposición de desastres propuesto por North (2004), en una población existen múltiples grupos de personas que responderán de manera distinta, según su grado de exposición a un evento. Este modelo explica hallazgos relacionados con las diferentes prevalencias asociadas al trastorno de estrés post-traumático (TEPT): entre $25 \%$ a $75 \%$ cuando la población está expuesta, entre 1\% y $11 \%$ cuando se encuentra protegida (Galea,
Nandi, \& Vlahov, 2005). En concreto, después de ocurrido un terremoto quienes perdieron su casa tienen más impacto que quienes no sufrieron esa pérdida (Leiva-Bianchi \& Araneda, 2013; LeivaBianchi \& Quintana, 2010; Sharan, Chaudhary, Kavathekar, \& Saxena, 1996).

Existen otros dos trabajos que incluyen el grado de exposición al evento como parte de su definición. Según Niitsu, Watanabe-Galloway, Sayles, Houfek y Rice (2014), el impacto psicológico se define mediante un modelo en el cual variables contextuales (factores socioculturales y la exposición) explican los síntomas de TEPT después de un desastre como el terremoto y tsunami de Tohoku, Japón, el 11 de marzo de 2011. Por su parte el metaanálisis de Leiva-Bianchi, Ahumada, Araneda y Botella (2018) propone dos grandes dimensiones independientes: salud-disrupción y exposiciónprotección. De su combinación, surgen cuatro tipos de IPS: (1) resiliente (i.e., responder de manera saludable cuando se está expuesto al evento); (2) traumático (i.e., responder disruptivamente cuando se está expuesto); (3) testigo (i.e., responder saludablemente cuando se está protegido); y (4) sensible (i.e., responder de manera disruptiva cuando se está protegido del evento; Figura 1).

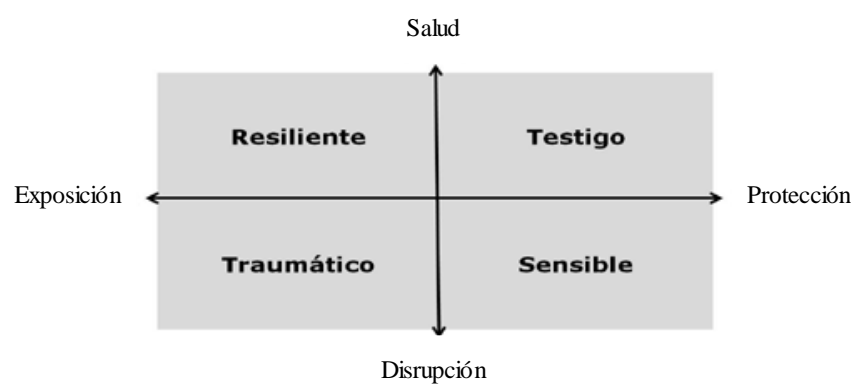

Figura 1. Modelo de impacto psicosocial o PSI. 
Combinar las dimensiones de respuesta con las de exposición es relevante, más aún para el caso de los desastres. Por ejemplo, 17 estudios sobre IPS de los desastres analizan variables saludables o disruptivas en función de factores de exposición-protección que explicarían parte de sus cambios (Tabla 1).

Tabla 1. Características de estudios que miden impacto psicosocial de los desastres.

\begin{tabular}{|c|c|c|c|c|}
\hline Autores & País & Evento & $\mathrm{N}$ & Media (DS) edad \\
\hline Amir \& Sol, 1999 & Israel & Guerra & 983 & $23.54(2.48)$ \\
\hline Belter et al., 1991 & USA & Huracán & 126 & $(--)$ \\
\hline Fischer et al., 2011 & Inglaterra & Terrorismo & 40 & $25.8(8.6)$ \\
\hline Freedy et al., 1992 & USA & Huracán & 418 & $40.2(10.4)$ \\
\hline Henriksen et al., 2010 & USA & Terrorismo & 34441 & $(--)$ \\
\hline Jia et al., 2010 & China & Terremoto & 276 & $67.8(5.9)$ \\
\hline Jones et al., 2002 & USA & Incendio & 22 & $9.4(1.6)$ \\
\hline Mels et al., 2010 & Congo & Guerra & 323 & $16(1.8)$ \\
\hline Misra et al., 2009 & Inglaterra & Terrorismo & 341 & 37 \\
\hline Paardekooper et al., 1999 & Sudan y Uganda & Guerra & 203 & $9.36(1.52)$ \\
\hline Palmieri et al., 2008 & Israel & Guerra & 1200 & $42.9(16.7)$ \\
\hline Shiri et al., 2008 & Israel & Guerra & 68 & 32.9 \\
\hline Sutker et al., 2002 & USA & Huracán & 206 & 33.2 \\
\hline Thompson et al., 2010 & USA & Huracán & 136 & $20.8(3.9)$ \\
\hline Ticehurst et al., 1996 & Australia & Terremoto & 3007 & 40 \\
\hline Viswanath et al., 2012 & India & Tsunami & 438 & $67.6(6.9)$ \\
\hline Weems et al., 2007 & USA & Huracán & 401 & 33 \\
\hline
\end{tabular}

Nota: el número total de estudios es 42629 
El presente estudio aporta a resolver esta controversia definiendo el IPS de los desastres, diseñando y validando la primera escala para medirlo reportada en la literatura científica de corriente principal. Para ello, se revisó literatura centrada en el IPS de los desastres. Dicha revisión mostró tres dimensiones: exposición-protección, procesamiento y respuestas. La primera, exposición-protección, se refiere al desastre como un EPT ambiental propiamente tal. Al respecto, se sabe que el EPT tiene distintas magnitudes (Bonanno, 2004; Bonanno et al., 2010) y que los grupos responden de forma diferente según el nivel de exposición (e.g., pérdida de la vivienda; Leiva-Bianchi \&
Araneda, 2013; Leiva-Bianchi \& Quintana, 2010; Niitsu et al., 2014; North, 2004; Sharan et al., 1996). Esto puede tener efectos en la prevalencia del TEPT (Galea et al., 2005). La segunda dimensión concibe a la persona como un organismo que procesa el EPT. Aquí se incluye el autoconcepto (positivo o negativo), auto-conexión, perdida de sentido y espiritualidad (Lai et al., 2012). Finalmente, la tercera dimensión se refiere a las respuestas de la persona frente al EPT. Los trabajos de Morgan y colaboradores (2001), Lai y colaboradores (2012) y Leiva-Bianchi y colaboradores (2018) clasifican las respuestas como disruptivas y saludables.

\section{MÉTODO}

Estudio piloto

La versión piloto de la escala de Impacto Psicosocial de Desastres (en inglés "Scale of Psychosocial Impact of Disasters" o SPSI-D) se diseñó con base en los instrumentos más frecuentemente utilizados en los estudios sobre desastres revisados en ISI Web of Knowledge que incluyeron en su título psychological impact o psychosocial impact (Tabla 1). Posteriormente se diseñaron 231 ítems agrupados en torno a tres dimensiones (exposición-protección, procesamiento y respuesta). El contenido de los ítems y las dimensiones fue evaluado por 6 jueces expertos en IPS, mediante el coeficiente de acuerdo inter-jueces (Cohen, 1968; Cohen, Swerdlik, \& Phillips, 1996; Dubé, 2008), del cual se obtuvieron 193 ítems con un coeficiente de acuerdo entre $.61 \mathrm{y} 1$.

Esta versión de la SPSI-D fue aplicada después de 5 años de ocurrido el terremoto y tsunami del 27 de febrero de 2010 (27-F) en la ciudad de Constitución, que fue parcialmente destruida por el tsunami posterior al terremoto, que alcanzó 8.8 grados Richter de intensidad. La escala fue aplicada a una muestra no probabilística de 467 habitantes de Constitución. Esta muestra fue dividida en dos. La primera consta de 237 personas, de las cuales el $61.2 \%$ tienen entre 18 y 44 años, $64.6 \%$ son mujeres y un $67.5 \%$ tuvieron daños en la vivienda. Con esta muestra se realizó el análisis factorial con extracción de factores usando análisis paralelo. Por su parte, la segunda muestra fue de 230 participantes, de 
los cuales $83.2 \%$ tienen entre 18 y 44 años, $62.5 \%$ son mujeres y un $53.4 \%$ tuvieron daños en la vivienda. Con esta muestra se realizó el análisis factorial confirmatorio (CFA). Los procedimientos e instrumento aplicado fueron aprobados por el Comité de Bioética de la Universidad de Talca.

\section{Análisis paralelo}

Para evaluar su validez se realizó un análisis paralelo con implementación óptima (Timmerman \& Lorenzo-Seva, 2011), método de extracción mínimos cuadrados no ponderados (ULS), rotación oblicua promin y considerando el percentil 95 para recomendar dimensiones (Lorenzo-Seva \& Van Ginkel, 2016). Para determinar la adecuación de la matriz de correlaciones, se utilizó la prueba de esfericidad de Bartlett $(p<.05)$ junto con el coeficiente Kaiser-Meyer-Olkin $(K M O \geq .8)$ y el porcentaje de varianza explicada (Kaiser, 1974). Finalmente, desde la matriz de factores rotados se eliminó aquellos ítems cuyos pesos factoriales $(w)$ fuesen menores a .40 en el factor donde más cargasen, que tuviesen cargas cruzadas menores a .30 en los demás factores y cuyas diferencias con los demás fuesen menores a .20 (Howard, 2015; Williams, Onsman, \& Brown, 2010). Se excluyeron aquellos ítems sin una relación consistente de significado entre las dimensiones y sus factores. Los análisis se realizaron con FACTOR versión 10. Este procedimiento cumple con las recomendaciones actuales para construir escalas mediante análisis factorial (Ferrando \& Lorenzo-Seva, 2014; Howard, 2015; Lloret-
Segura, Ferreres-Traver, Hernández-Baeza, \& Tomás-Marco, 2014).

Análisis factorial confirmatorio

Se realizó un CFA usando estimación por el método de máxima verosimilitud. Se consideró que la escala es válida cuando los indicadores de ajuste estén dentro de los siguientes límites: $C M I N / D F<3, R M S E A<.08, N F I>.9, C F I>.9$ y PNFI> .5 (Barret, 2007; Hair, Black, Babin, \& Anderson, 2014). Además, se eliminaron aquellos ítems y dimensiones de la SPSI-D que no tuviesen pesos significativos $(p>.05)$ y su efecto fuese pequeño ( $w<.4$; Howard, 2015). Para estos análisis se utilizó AMOS 16.

Fiabilidad, validez de criterio, sensibilidad y especificidad

A partir de ambas muestras en conjunto se calculó la fiabilidad de la SPSI-D y sus dimensiones mediante el estadístico Alpha de Cronbach ( $\alpha>.7$; Mislevy \& Bock, 1990). También se evaluó la validez de criterio de la escala y sus dimensiones mediante la relevancia $(r>.4)$ y significación estadística $(p<.05)$ de la estructura de correlaciones (Pardo, 2014; Taylor, 1990) con dos variables: los síntomas de TEPT y la respuesta de crecimiento post-traumático (en inglés post-traumatic growth o PTG). Para evaluar la sintomatología se utilizó la Short Post-traumatic Stress Disorder Rating Interview (SPRINT-E; Leiva-Bianchi \& Gallardo, 2013; Norris, Hamblen, Brown, \& Schinka, 2008). Cada pregunta posee una escala de intensidad de 
0 (mínima) a 4 (máxima), excepto la 12 que es dicotómica $(0=$ sin ideación y $1=$ con ideación suicida). Si la respuesta a una pregunta es mayor o igual a 3 (1 para el ítem 12), se considera un síntoma intenso. Un total de 3 o más síntomas intensos indica un caso de TEPT (sobre 7, la probabilidad de un falso positivo es muy baja; (Norris et al., 2008). Por su parte, se utilizó el Post-traumaitc Growth Inventory (PTGI) para evaluar PTG. Un puntaje total de PTGI mayor que 50 puntos indica un caso de crecimiento personal (Leiva-Bianchi \& Araneda, 2014).

Finalmente, se evaluó la precisión de los instrumentos comparando el total de la SPSI-D y sus dimensiones con los casos de TEPT y PTG reportados por SPRINT-E y PTGI mediante curvas ROC $(A U C>.8 ; p<.05)$. Este análisis también permite identificar los puntos de corte para el total y sus dimensiones. Bajo el supuesto que la SPSI-D debía ser sensible (Sensibilidad > .600) para detectar casos de TEPT y específica $($ Falso-positivo < .10) para detectar casos de PTG, se utilizó puntos de corte que dieran una baja probabilidad de falsos positivos en desmedro de la sensibilidad de la medida. Esto, porque diagnosticar a alguien con TEPT cuando probablemente no lo padezca implica darle una etiqueta diagnóstica que puede estigmatizarle. Con el PTG ocurre lo contrario, es mejor contar con una medida sensible porque declarar que alguien ha crecido como persona cuando en realidad no, probablemente no sea iatrogénico. Se dejó a disposición del personal de salud esta versión de la SPSI-D en un formato listo para ser aplicado (Ver anexos). Estos análisis fueron realizados con SPSS versión 14 .

\section{RESULTADOS}

Evaluación de la dimensionalidad

Después de aplicada la escala validada por los jueces-expertos a la muestra de 237 personas, a través del análisis paralelo, se encontró una solución admisible $\left(K M O=.835 ; X^{2}=25309.6\right.$; $p<.001)$ que explica un $51.1 \%$ de la varianza en torno a 8 factores (ver en https://www.dropbox.com/s/l6ybvgkyb154q2s/ex ploratorio.xlsx?dl=0). Utilizando la matriz de factores rotados y aplicando la regla de Howard (2015), la SPSI-D se redujo a 63 ítems y 6 dimensiones: exposición; apoyo social; creencias de suerte; disrupción; salud; y autoconcepto positivo.

Confirmación de dimensiones

Con base en ese modelo y en la submuestra de 230 personas, se determinó la validez de constructo de la escala mediante CFA. Después de quitar 12 ítems cuyo peso fue menor que .4, se obtuvieron niveles adecuados ajustes $(C M I N / D F=2.508 ; \quad R M S E A=.081) \quad$ y $\quad \mathrm{de}$ parsimonia $(P N F I=.595)$ lo que no ocurrió con los ajustes incrementales $(N F I=.623$; $C F I=.731)$. Además, todos los parámetros 

Amaya I., Antúnez S., ... Serrano, C. (2019). Diseño y validación de una escala de impacto psicosocial de los desastres (SPSI-D). Revista de Sociología, 34(1), 21-42. doi: 10.5354/0719-529X.2019.53141

fueron relevantes $(w>.4)$ y significativos $(p<.05)$. La versión finalmente obtenida de la
SPSI-D quedó conformada por 51 ítems agrupados en 6 dimensiones (Figura 2).

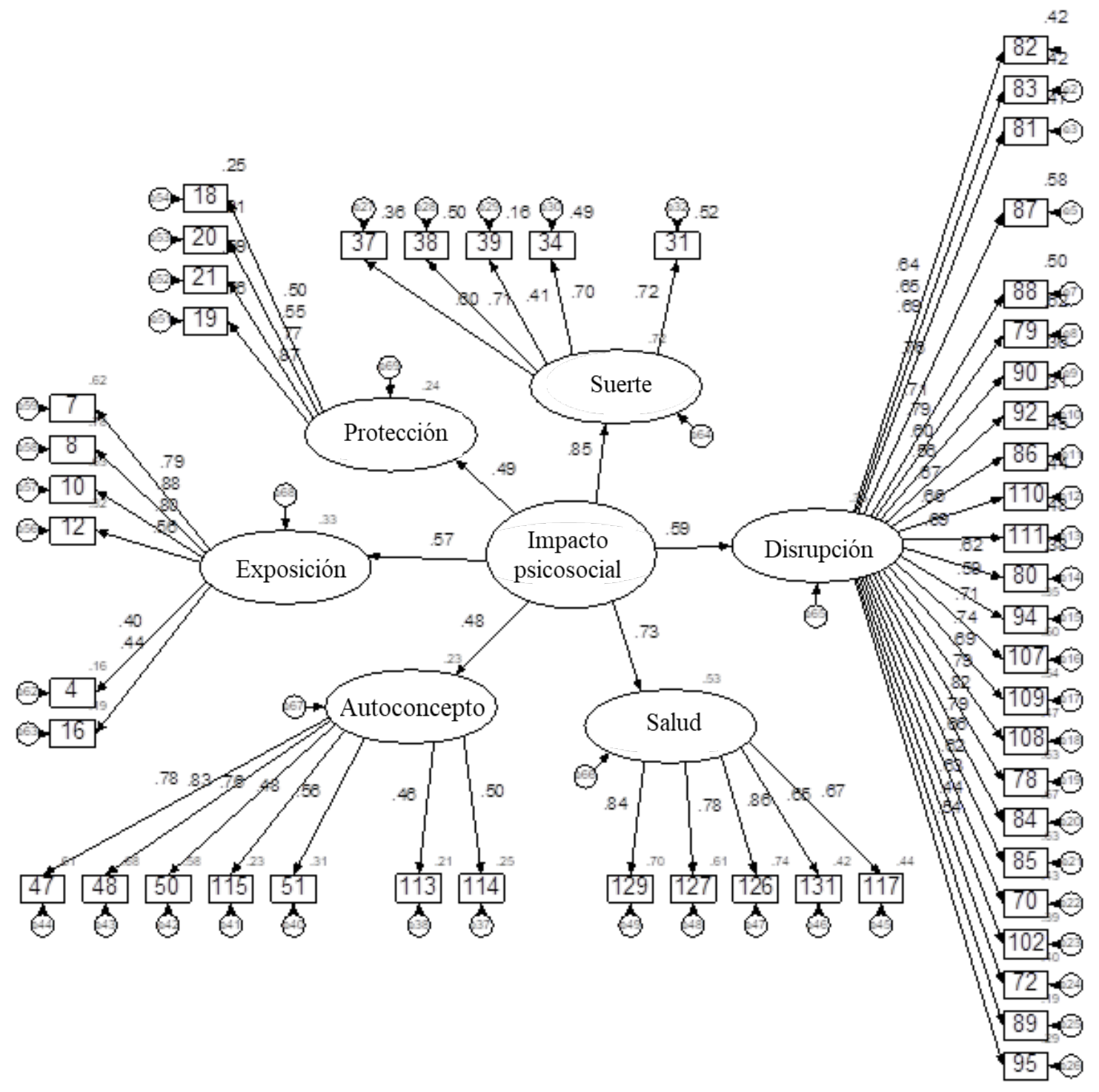

Figura 2. Modelo de SPSI-D.

Fiabilidad, validez de criterio, sensibilidad y especificidad

La SPSI-D tuvo muy buenos indicadores de fiabilidad, tanto en el total de puntuaciones $(\alpha=.942)$, como en sus dimensiones. Asimismo, posee correlaciones relevantes y significativas con el total de síntomas intensos de TEPT allí donde es esperable que existan esas correlaciones. Aumentan los síntomas en la 
medida que aumentan el total de la SPSI-D $(r=.600 ; p<.001)$, las respuestas disruptivas $(r=.610 ; p<.001)$ y la exposición $(r=.404$; $p<.001)$. Del resto de las correlaciones, 3 no fueron relevantes y una tampoco significativas (Tabla 2)

Tabla 2. Fiabilidad, asimetría, curtosis de la SPSI-D, correlaciones significativas con los síntomas intensos de PTSD y PTG, sensibilidad, falsos positivos y puntos de corte.

\begin{tabular}{lccccccccc}
\multicolumn{1}{c}{ SPSI-D } & $\boldsymbol{\alpha}$ & $\boldsymbol{S} \boldsymbol{k}$ & $\boldsymbol{K u}$ & $\begin{array}{c}\text { PTSD } \\
(\boldsymbol{r})\end{array}$ & $\begin{array}{c}\text { PTG } \\
(\boldsymbol{r})\end{array}$ & $\boldsymbol{A U C}$ & Sensibilidad & $\begin{array}{c}\text { Falsos } \\
\text { positivos }\end{array}$ & $\begin{array}{c}\text { Puntos } \\
\text { de } \\
\text { corte }\end{array}$ \\
\hline Exposición & .826 & 0.447 & -1.018 & $.404^{*}$ & $.321^{*}$ & $.757^{*}$ & .403 & .095 & 12 \\
$\begin{array}{l}\text { Apoyo social } \\
\text { Creencias de }\end{array}$ & .761 & 0.915 & 0.010 & $.141^{*}$ & $.238^{*}$ & $.586^{*}$ & .227 & .074 & 6 \\
suerte & .759 & 0.062 & -1.012 & $.354^{*}$ & $.567^{*}$ & $.775^{*}$ & .573 & .162 & 8 \\
Autoconcepto & & & & & & & & & \\
positivo & .825 & -1.026 & 1.430 & .086 & $.415^{*}$ & $.685^{*}$ & .641 & .346 & 16 \\
Salud & .873 & -0.810 & -0.103 & $.284^{*}$ & $.734^{*}$ & $.851^{*}$ & .621 & .140 & 11 \\
$\begin{array}{l}\text { Disrupción } \\
\text { Total }\end{array}$ & .954 & -0.934 & -0.033 & $.610^{*}$ & $.389^{*}$ & $.865^{*}$ & .649 & .087 & 34 \\
\hline
\end{tabular}

Nota: SPSI-D = Impacto Psicosocial de Desastres. $\alpha=$ fiabilidad medida a través de Alpha de Cronbach. Sk $=$ Asimetría. $\mathrm{Ku}=$ Curtosis. $\mathrm{PTSD}=$ Estrés postraumático. $\mathrm{PTG}=$ crecimiento post-traumático. $(\mathrm{r})=$ correlación de Pearson. AUC = área bajo la curva (en inglés "area under curve"), mientras más cercano a 1, mayor precisión. Aplicada al total de la SPSI-D y al de cada una de las dimensiones, la prueba Kolmogorov-Smirnov indica que los totales se distribuyen normalmente $(p<.01) .{ }^{*}$ Estadísticos significativos $(p<.01)$.

Con base en las correlaciones relevantes y significativas, se analizó su sensibilidad y especificidad para detectar casos de PTSD mediante curvas ROC. Una persona con TEPT tendrá una puntuación mayor en la escala que otra escogida al azar entre quienes no tienen PTSD un $88.6 \%$ de las veces para el total de SPSI-D $(A U C=.886 ; p<.001)$, un $86.5 \%$ para la dimensión disrupción $(A U C=.865 ; p<.001) \mathrm{y}$ un $75.7 \%$ de las veces para la dimensión exposición $(A U C=.757 ; p<.001)$. Los puntos de corte que minimizaron la probabilidad de encontrar un falso positivo de TEPT fueron 90 puntos para el total de SPSI-D (Sensibilidad $=.662 ; \quad$ Falso-positivo $=.095), 34$ puntos para la dimensión disrupción (Sensibilidad $=.649 ;$ Falso-positivo $=.087)$ y 12 puntos para exposición (Sensibilidad =.403; Falso-positivo $=$.095) (Figura 3$)$. 


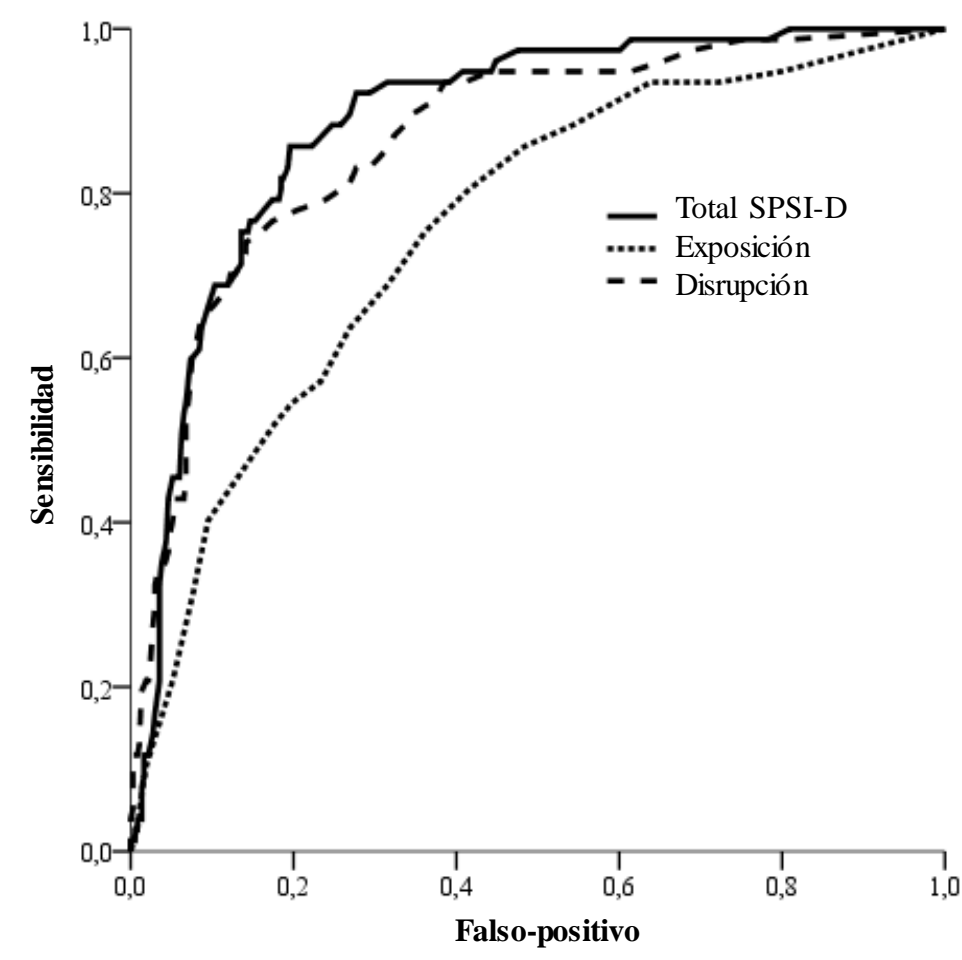

Figura 3. Sensibilidad y falsos positivos para total de SPSID, exposición y disrupción. Los segmentos diagonales indican empates.

Respecto de la validez de criterio con PTG, nuevamente la SPSI-D presentó correlaciones relevantes y significativas allí donde se esperaba que las hubiese. Aumenta el crecimiento en la medida que aumenta el total de la SPSI-D $(r=.599)$, las respuestas saludables $(r=.734)$, el autoconcepto positivo $(r=.415)$ y las creencias de suerte $(r=.567)$.

Como ya fue usado el total de SPSI-D en el análisis de la sensibilidad y especificidad anterior, fue excluido para detectar casos de PTG. En su reemplazo y por presentar una mayor correlación con PTG que con PTSD, se incluyó la dimensión apoyo social. Una persona con PTG tendrá una puntuación mayor en la escala que otra escogida al azar entre quienes no han crecido un $85.1 \%$ de las veces para respuestas saludables $(A U C=.851 ; p<.001)$, un $68.5 \%$ para la dimensión autoconcepto positivo $(A U C=.685 ; p<.001)$, un $77.5 \%$ para creencias de suerte $(A U C=.775 ; p<.001)$ y un $58.6 \%$ de las veces para apoyo social $(A U C=.586$; $p<.001)$. Los puntos de corte fueron 11 para salud (Sensibilidad $=.621 ;$ Falso-positivo $=.140$ ), 16 puntos para autoconcepto positivo $\left(\right.$ Sensibilidad $=.641 ; \quad$ Falso ${ }^{-}$positivo $\left.=.346\right), 8$ puntos para creencias de suerte $($ Sensibilidad $=.573 ;$ Falso-positivo $=.162)$ y 6 puntos para apoyo social (Sensibilidad $=.227$; Falso-positivo $=.074 ;$ Figura 4 ) . 


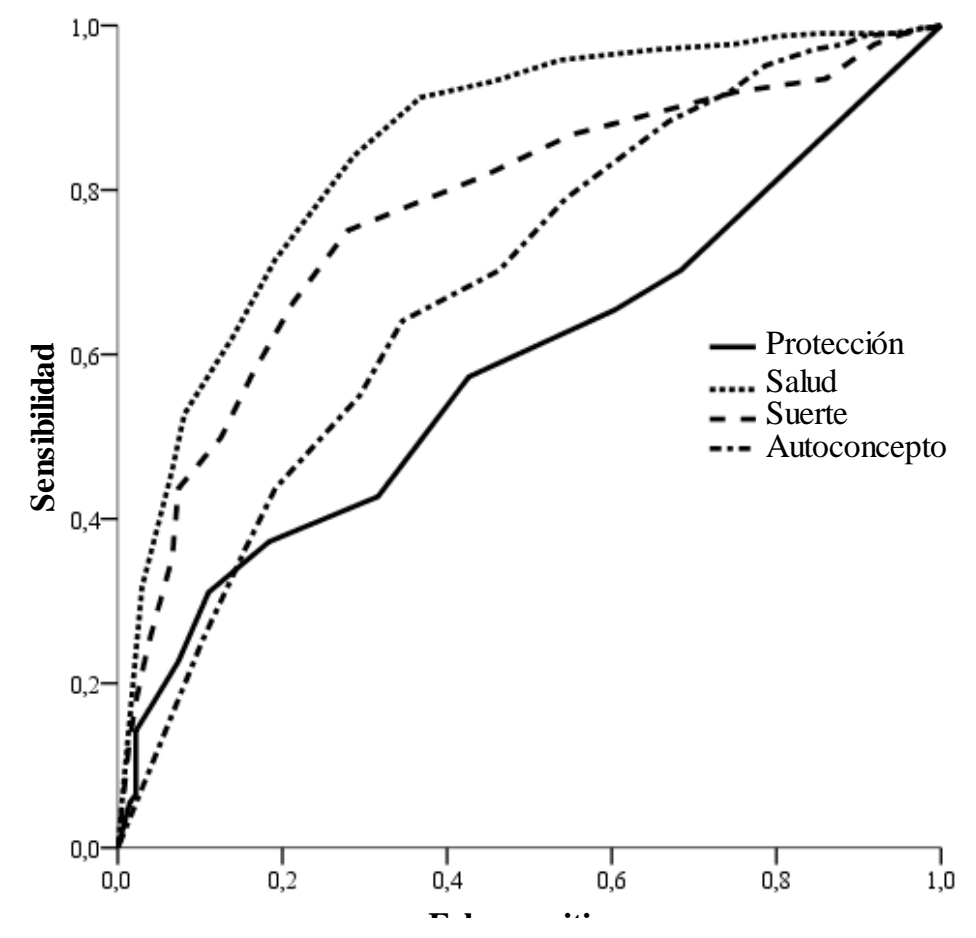

Figura 4. Sensibilidad y falsos positivos para las dimensiones protección, salud, suerte y autoconcepto. Los segmentos diagonales indican empates.

\section{CONCLUSIONES}

Este estudio sustenta de manera empírica el concepto de Impacto Psicosocial, ya utilizado para otros eventos, como atentados terroristas (Morgan et al., 2011), cáncer (Lai et al., 2012) o desastres naturales (Leiva-Bianchi et al., 2018). En concreto, con sus 51 ítems agrupados en 6 dimensiones, la SPSI-D cumple con los criterios de validez, confiabilidad y precisión para dar cuenta de la definición operacional del concepto. Dada su validez de criterio, esta escala integra las ventajas de tener los instrumentos que miden respuestas disruptivas (e.g., estrés post- traumático; Leiva-Bianchi \& Gallardo, 2013) y saludables (e.g., crecimiento post-traumático; Leiva-Bianchi \& Araneda, 2014) después de un desastre.

Además de lo anterior, la SPSI-D permite evaluar ambos tipos de respuesta en función de la exposición y protección (dimensión apoyo social) ante el evento. Esto resuelve controversias relacionadas con las diferencias en las prevalencias de respuestas disruptivas después de un evento potencialmente traumático (e.g., desastres), solamente porque no se 
considera la exposición al mismo. Se espera que entre un $10 \%$ y $60 \%$ respondan negativamente después de dos años (Bonanno \& Diminich, 2013), proporción que se mantiene en el tiempo (Crawford et al., 2010; Dekel, Ein-Dor, \& Solomon, 2012; Galea et al., 2005; Green et al., 1990; Holgersen, Boe, \& Holen, 2010; Lazaratou et al., 2008). Sin embargo, estas proporciones varían bastante, en torno al 40\%. Esto es un problema epidemiológico importante porque dificulta las comparaciones entre estudios, entre eventos y entre distintos momentos de medición. Es decir, se combinan personas con alta exposición con personas más protegidas (Galea et al., 2005). De hecho, las proporciones de respuestas disruptivas son mayores entre quienes están expuestos, $25 \%$ a $75 \%$; y menores entre los protegidos, 1\% a 11\% (Galea et al., 2005). De ahí la importancia de incorporar la exposición-protección a cualquier constructo que intente medir este tipo de eventos. Y, por supuesto, a las políticas públicas que se desprendan de dichas mediciones.

La SPSI-D presenta otra característica destacable. Incluye dos dimensiones relacionadas con las creencias que las personas podrían tener después de un desastre. Es decir, las creencias de suerte, religiosas y las vinculadas al autoconcepto. Dichas creencias están a la base del procesamiento de los EPT y de la capacidad que cada persona tiene de incorporarlas en su memoria verbalmente accesible o conciencia (Ehring et al., 2014; Peres, McFarlane, Nasello, \& Moores, 2008). Este procesamiento personal es relevante para que las comunidades puedan recuperarse después desastres.

Ahora bien, como se señaló, las dimensiones de la SPSI-D se basaron en el impacto psicosocial de eventos médicos y no médicos (desastres) operacionalizados por los modelos como variables positivas y negativas. Entendemos que estos aspectos están moderados por el grado de exposición o protección al evento, el procesamiento cognitivo de las personas y su correspondiente respuesta disruptiva o saludable, desde un paradigma neurocognitivo (Leiva-Bianchi et al., 2018). La escala cuenta con seis dimensiones, alguna de ellas como exposición, respuesta de salud y disruptiva se desprenden de los modelos mencionados (Lai et al., 2012; Morgan et al., 2011; North, 2004); en cambio otras, como apoyo social (protección) y procesamiento (creencias y autoconcepto) requieren mayor análisis.

Una posible explicación de que la dimensión teórica de protección se entendiera principalmente como apoyo social se debería a la importancia crucial del apoyo recibido y percibido para generar diferentes patrones de ajuste en las personas, posterior a un desastre (Bonanno et al., 2010). Estas diferencias en el apoyo estarían moderadas por aspectos relacionados con la comorbilidad, edad, género y recursos económicos asociados con la muestra (Bonanno \& Diminich, 2013; Litz, Gray, Bryant, \& Adler, 2002). Es importante destacar diferencias en las respuestas al trauma en el contexto chileno, ya que ayudaría a entender en cierta medida su variabilidad en las personas expuestas a desastres (Weems et al., 2007) 
Respecto a las creencias, entendemos que los desastres generan cambios positivos y negativos en las personas, respecto a sí mismas, los otros y el mundo (Leiva-Bianchi, Baher, \& Poblete, 2012). Como indican Nogami y Yoshida (2014), luego de un desastre es común que se generen creencias sobre los patrones de comportamiento de las personas, pero sin contrastarlo con la realidad, por lo que pudieran ser falsas. Estos autores hipotetizan que las creencias luego de un desastre se ven moduladas por las diversas fuentes de información del evento, clasificándolas en públicas (e.g., televisión, internet) y privadas (e.g., familiar, amigos, vecinos). Pensamos que las creencias de suerte, sí mismo (autoconcepto) y religiosas que emergen de la escala pudieran comprenderse, en parte, por esta influencia. Estas creencias ayudarían a las personas a interpretar el evento, percibir el riesgo potencial del desastre y su capacidad para enfrentarlo (Inal \& Dogan, 2018; Sun, Deng, \& Qi, 2018).

Con todo, la escala diseñada abordaría de manera satisfactoria las dimensiones del modelo de impacto psicosocial (Leiva-Bianchi et al., 2018), a través de un paradigma que incorpora el estímulo (niveles de exposición-protección al evento), organismo (procesamiento cognitivo, creencias) y respuesta (disruptivas y saludables). Sin embargo, llama la atención la cantidad de ítems que tiene la dimensión disrupción, lo que puede deberse a las características negativas que los desastres tienen para las personas (Davis, et al., 2010; Jia et al., 2010; Sutker, et al., 2002; Viswanath et al., 2012; Weems et al., 2007).

Por último, Chile pierde cerca del $1 \%$ de su Producto Interno Bruto anual producto de desastres, lo cual ha orientado políticas públicas de los últimos años (Consejo Nacional de Innovación para el Desarrollo [CNID], 2016). En este marco, el uso de instrumentos válidos, fiables y precisos como la SPSI-D es de crucial relevancia para diagnosticar a las personas insertas en comunidades y realizar intervenciones cuyo efecto pueda ser comprobado. Para facilitar esta evaluación, se deja a libre disposición de los profesionales la SPSI-D en un formato listo para ser aplicado e interpretado (Anexo 1).

Finalmente, una limitación del estudio es la estimación por máxima verosimilitud utilizada en el CFA. Debido a que los ítems de la SPSI-D son ordinales y probablemente no se distribuyan normalmente, correspondería utilizar un método robusto para estas variables, como mínimos cuadrados no ponderados (Satorra \& Bentler, 1994). También podría utilizarse máxima verosimilitud y boostraping (Bollen \& Stine, 1990). Sugerimos incorporar este tipo de análisis en futuras validaciones.

\section{REFERENCIAS}

Amir, M., \& Sol, O. (1999). Psychological impact and prevalence of traumatic events in a student sample in Israel: The effect of multiple traumatic events and physical injury. Journal of Traumatic Stress, 12(1), 139-154. doi: 10.1023/A:1024754618063

Barret, P. (2007). Structural equation modelling: Adjudging model fit. Personality and 
Individual Differences, 42(5), 815-824. doi: 10.1016/j.paid.2006.09.018

Belter, R. W., Dunn, S. E., \& Jeney, P. (1991). The psychological impact of the Hurricane Hugo on children: A needs assessment. Advances in Behaviour Research and Therapy, 13(3), 155-161. doi: 10.1016/01466402(91)90003-S

Bollen, K. A., \& Stine, R. (1990). Direct and indirect effects: Classical and bootstrap estimates of variability. Sociological Methodology, 20(1), 115-140. doi: $10.2307 / 271084$

Bonanno, G. A. (2004). Loss, trauma, and human resilience: Have we underestimated the human capacity to thrive after extremely aversive events? The American Psychologist, 59(1), 20-28. doi: 10.1037/0003-066X.59.1.20

Bonanno, G. A., Brewin, C. R., Kaniasty, K., \& Greca, A. M. L. (2010). Weighing the costs of disaster: Consequences, risks, and resilience in individuals, families, and communities. Psychological Science in the Public Interest, 11(1), 1-49. doi: 10.1177/1529100610387086

Bonanno, G. A., \& Diminich, E. D. (2013). Annual research review: Positive adjustment to adversity - trajectories of minimal - impact resilience and emergent resilience. Journal of Child Psychology and Psychiatry, 54(4), 378-401. doi: 10.1177/1529100610387086

Consejo Nacional de Innovación para el Desarrollo. (2016). Hacia un Chile resiliente frente a desastres: Una oportunidad. Recuperado de http://www.cnid.cl/wpcontent/uploads/2016/12/INFORME-

DESASTRES-NATURALES.pdf

Cohen, J. (1968). Weighted kappa: Nominal scale agreement provision for scaled disagreement or partial credit. Psychological Bulletin, $\quad 70(4), \quad 213-220 . \quad$ doi: 10.1037/h0026256

Cohen, R. J., Swerdlik, M. E., \& Phillips, S. M. (1996). Psychological testing and assessment: An introduction to tests and measurement. Londres, Reino Unido: Mayfield Publishing Co.

Crawford, A. V., Green, S. B., Levy, R., Lo, W. J., Scott, L., Svetina, D., \& Thompson, M. S. (2010). Evaluation of parallel analysis methods for determining the number of factors. Educational and Psychological Measurement, 70(6), 885-901. doi: 10.1177/0013164410379332

Davis, T. E., Grills-Taquechel, A. E., \& Ollendick, T. H. (2010). The psychological impact from Hurricane Katrina: Effects of displacement and trauma exposure on university students. Behavior Therapy, 41(3), 340-349. doi: 10.1016/j.beth.2009.09.004

Dekel, S., Ein-Dor, T., \& Solomon, Z. (2012). Posttraumatic growth and posttraumatic distress: A longitudinal study. Psychological Trauma: Theory, Research, Practice, and Policy, 4(1), 94-101. doi: 10.1037/a0021865

Dubé, É. (2008). Evaluación del acuerdo interjueces en investigacion clinica: Breve introducción a la confiabilidad interjueces. Revista Argentina de Clínica Psicológica, 18(1), 75-80.

Ehring, T., Welboren, R., Morina, N., Wicherts, J. M., Freitag, J., \& Emmelkamp, P. M. G. (2014). Meta-analysis of psychological treatments for posttraumatic stress disorder in adult survivors of childhood abuse. Clinical Psychology Review, 34(8), 645-657. doi: 10.1016/j.cpr.2014.10.004

Ferrando, P. J., \& Lorenzo-Seva, U. (2014). El análisis factorial exploratorio de los ítems: Algunas consideraciones adicionales. Anales de Psicología, 30(3), 1170-1175. doi: 10.6018/analesps.30.3.199991

Fischer, P., Postmes, T., Koeppl, J., Conway, L., \& Fredriksson, T. (2011). The meaning of collective terrorist threat: Understanding the subjective causes of terrorism reduces its negative psychological impact. Journal of 
Interpersonal Violence, 26(7), 1432-1445. doi: 10.1177/0886260510369137

Freedy, J. R., Shaw, D. L., Jarrell, M. P., \& Masters, C. R. (1992). Towards an understanding of the psychological impact of natural disasters: An application of the conservation resources stress model. Journal of Traumatic Stress, 5(3), 441-454. doi: 10.1007/BF00977238

Galea, S., Nandi, A., \& Vlahov, D. (2005). The epidemiology of post-traumatic stress disorder after disasters. Epidemiologic Reviews, 27(1), 78-91. doi: 10.1093/epirev/mxi003

Green, B. L., Lindy, J. D., Grace, M. C., Gleser, G. C., Leonard, A. C., Korol, M., \& Winget, C. (1990). Buffalo Creek survivors in the second decade: Stability of stress symptoms. American Journal of Orthopsychiatry, 60(1), 43-54. doi: 10.1037/h0079168

Guha-Sapir, D., Hargitt, D., \& Hoyois, P. (2004). Thirty years of natural disasters 1974-2003. The numbers. Lovaina, Bélgica: Presses Universitaires de Louvain

Guha-Sapir, D., Hoyois, P., \& Below, R. (2014). Annual disaster statistical review 2014 - The numbers and trends. Lovaina, Bélgica: Ciaco Imprimerie

Hair, J. F., Black, W. C., Babin, B. J., \& Anderson, R. E. (2014). Multivariate data analysis. Londres, Reino Unido: Pearson Education Limited.

Henriksen, C., Bolton, J. M., \& Sareen, J. (2010). The psychological impact of terrorist attacks: Examining a dose-response relationship between exposure to 9/11 and axis I mental disorders. Depression and Anxiety, 27(11), 993-1000. doi: 10.1002/da.20742

Hobfoll, S. E. (1989). Conservation of resources. A new attempt at conceptualizing stress. The American Psychologist, 44(3), 513-524. doi: 10.1037//0003-066X.44.3.513

Hobfoll, S. E. (2012). Conservation of resources and disaster in cultural context: The caravans and passageways for resources. Psychiatry: Interpersonal \& Biological Processes, 75(3), 227-233. doi: 10.1521/psyc.2012.75.3.227

Holgersen, K. H., Boe, H. J., \& Holen, A. (2010). Long-term perspectives on posttraumatic growth in disaster survivors, 23(3), 413-416. doi: $10.1002 /$ jts.

Howard, M. (2015). A review of exploratory factor analysis (EFA). Decisions and overview of current practices: What we are doing and how can we improve? International Journal of Human-Computer Interaction, $\quad 32(1), \quad 51-62, \quad$ doi: 10.1080/10447318.2015.1087664

Inal, E., \& Dogan, N. (2018). Improvement of general disaster preparedness belief scale based on health belief model. Prehospital and Disaster Medicine, 33(6), 627-636. doi: 10.1017/S1049023X18001012

Jia, Z., Tian, W., Liu, W., Cao, Y., Yan, J., \& Shun, Z. (2010). Are the elderly more vulnerable to psychological impact of natural disaster? A population-based survey of adult survivors of the 2008 Sichuan earthquake. BMC Public Health, 1O(172), 111. doi: 10.1186/1471-2458-10-172

Jones, R. T., Ribbe, D. P., Cunningham, P. B., Weddle, J. D., \& Langley, A. K. (2002). Psychological impact of fire disaster on children and their parents. Behavior Modification, 26(2), 163-186. doi: 10.1177/0145445502026002003

Kaiser, H. F. (1974). An index of factorial simplicity. Psychometrika, 39(1), 31-36. doi: 10.1007/BF02291575

Lai, J. S., Garcia, S. F., Salsman, J. M., Rosenbloom, S., \& Cella, D. (2012). The psychosocial impact of cancer: Evidence in support of independent general positive and negative components. Quality of Life Research: An International Journal of Quality of Life Aspects of Treatment, Care and Rehabilitation, 21(2), 195-207. doi: 10.1007/s11136-011-9935-2 
Lazaratou, H., Paparrigopoulos, T., Galanos, G., Psarros, C., Dikeos, D., \& Soldatos, C. (2008). The psychological impact of a catastrophic earthquake: A retrospective study 50 years after the event. The Journal of Nervous and Mental Disease, 196(4), 340344. doi: NMD.0b013e31816a62c6

Leaning, J., \& Guha-Sapir, D. (2013). Natural disasters, armed conflict, and public health. New England Journal of Medicine, 369(19), 1836-1842. doi: 10.1056/NEJMra1109877

Leiva-Bianchi, M., Ahumada, F., Araneda, A., \& Botella, J. (2018). What is the psychosocial impact of disasters? A meta-analysis. Issues in Mental Health Nursing, 39(4), 320-327. doi: 10.1080/01612840.2017.1393033

Leiva-Bianchi, M., \& Araneda, A. (2013). Prevalencia y sintomatología del estrés post traumático en personas que experimentan un terremoto y un tsunami. Salud \& Sociedad, 4(2), 146-155. doi: 10.22199/S07187475.2013.0002.00003

Leiva-Bianchi, M., \& Araneda, C. (2014). Confirmatory factor analysis of the posttraumatic growth inventory after Chilean earthquake. Journal of Loss and Trauma, 20(4), 1-9. doi: 10.1080/15325024.2013.873223

Leiva-Bianchi, M., Baher, G., \& Poblete, C. (2012). The effects of stress coping strategies in post-traumatic stress symptoms among earthquake survivors. An explanatory model of post-traumatic stress. Terapia Psicológica, 30(2), 51-59. doi: 10.4067/S0718-48082012000200005

Leiva-Bianchi, M. C., \& Gallardo, I. (2013). Validation of the short posttraumatic stress disorder rating interview (SPRINT-E) in a sample of people affected by F-27 Chilean earthquake and tsunami. Anales de Psicología, 29(2), 328-334. doi: 10.6018/analesps.29.2.130681

Leiva-Bianchi, M., \& Quintana, G. R. (2010). Factores ambientales y psicosociales vinculados a síntomas de ataque de pánico después del terremoto y tsunami del 27 de febrero de 2010 en la zona central de Chile. Terapia Psicológica, 28(2), 161-168. doi: 10.4067/S0718-48082010000200004

Litz, B. T., Gray, M. J., Bryant, R., \& Adler, A. B. (2002). Early intervention for trauma: Current status and future directions. Clinical Psychology: Science and Practice, 9(2), 112-134. doi: 10.1093/clipsy.9.2.112

Lloret-Segura, S., Ferreres-Traver, A., Hernández-Baeza, A., \& Tomás-Marco, I. (2014). El análisis factorial exploratorio de los ítems: Una guía práctica, revisada y actualizada. Anales de Psicología, 30(3), 1151-1169. doi: 10.6018/analesps.30.3.199361

Lorenzo-Seva, U., \& Van Ginkel, J. R. (2016). Multiple imputation of missing values in exploratory factor analysis of multidimensional scales: Estimating latent trait scores. Anales de Psicología, 32(2), 596608. doi: 10.6018/analesps.32.2.215161

Mels, C., Derluyn, I., Broekaert, E., \& Rosseel, Y. (2010). The psychological impact of forced displacement and related risk factors on eastern Congolese adolescents affected by war. Journal of Child Psychology and Psychiatry, and Allied Disciplines, 51(10), 1096-1104. doi: 10.1111/j.14697610.2010.02241.x

Mislevy, R. J., \& Bock, R. D. (1990). BILOG 3: Item analysis and test scoring with binary logistic models. Mooresville, NC: Scientific Software International.

Misra, M., Greenberg, N., Hutchinson, C., Brain, A., \& Glozier, N. (2009). Psychological impact upon London ambulance service of the 2005 bombings. Ocupational Medicine, 59(6), 428-433. doi: 10.1093/occmed/kqp100

Morgan, G. S., Wisneski, D. C., \& Skitka, L. J. (2011). The expulsion from Disneyland: The social psychological impact of 9/11. The American Psychologist, 66(6), 447-454. doi: 10.1037/a0024772 
Niitsu, K., Watanabe-Galloway, S., Sayles, H., Houfek, J., \& Rice, M. (2014). A pilot study of the psychological impact of the great east Japan earthquake and tsunami. Journal of the American Psychiatric Nurses Association, 20(3), 194-202. doi: 10.1177/1078390314536615

Nogami, T., \& Yoshida, F. (2014). Disaster myths after the great east Japan disaster and the effects of information sources on belief in such myths. Disasters, 38(2), 190-205. doi: 10.1111/disa. 12073

Norris, F., Hamblen, J., Brown, L., \& Schinka, J. (2008). Validation of the short posttraumatic stress disorder rating interview (expanded version, SPRINT-E) as a measure of postdisaster distress and treatment need. American Journal of Disaster Medicine, 3(4), 201-212.

North, C. S. (2004). Approaching disaster mental health research after the 9/11 world trade center terrorist attacks. Psychiatric Clinics of North America, 27(3), 589-602. doi: 10.1016/j.psc.2004.03.001

Paardekooper, B., De Jong, J. T., \& Hermanns, J. M. A. (1999). The psychological impact of war and the refugee situation on South Sudanese children in refugee camps in Northern Uganda: An exploratory study. Journal of Child Psychology and Psychiatry, 40(4), 529-536. doi: 10.1111/14697610.00471

Palmieri, P. A., Canetti-Nisim, D., Galea, S., Johnson, R., \& Hobfoll, S. E. (2008). The psychological impact of the Israel-Hezbollah war on Jews and Arabs in Israel: The impact of risk and resilience factors. Social Science \& Medicine, 67(8), 1208-1216. doi: 10.1016/j.socscimed.2008.06.030

Pardo, A. (2014). Análisis de datos en ciencias sociales y de la salud I. Madrid, España: Síntesis.

Peres, J. F. P., McFarlane, A., Nasello, A. G., \& Moores, K. (2008). Traumatic memories: bridging the gap between functional neuroimaging and psychotherapy. Australian and New Zealand Journal of Psychiatry, 42(6), 478-488. doi: 10.1080/00048670802050561

Plutchik, R. E., \& Conte, H. R. (1997). Circumplex models of personality and emotions. Washington, DC: American Psychological Association.

Russell, J. A. (2003). Core affect and the psychological construction of emotion. Psychological Review, 110(1), 145-172. doi: 10.1037/0033-295X.110.1.145

Satorra, A., \& Bentler, P. M. (1994). Corrections to test statistics and standard errors in covariance structure analysis. En A. von Eye \& C. C. Clogg (Eds.), Latent variables analysis: Applications for developmental research (pp. 399-419). Thousand Oaks, CA: Sage Publications, Inc.

Sharan, P., Chaudhary, G., Kavathekar, S., \& Saxena, S. (1996). Preliminary report of psychiatric disorders in survivors of a severe earthquake. The American Journal of Psychiatry, 153(4), 556-558. doi: 10.1176/ajp.153.4.556

Shiri, S., Wexler, I. D., Alkalay, Y., Meiner, Z., \& Kreitler, S. (2008). Positive and negative psychological impact after secondary exposure to politically motivated violence among body handlers and rehabilitation workers. The Journal of Nervous and Mental Disease, 196(12), 906-911. doi: 10.1097/NMD.0b013e31818ec80b

Sun, L., Deng, Y., \& Qi, W. (2018). Two impact pathways from religious belief to public disaster response: Findings from a literature review. International Journal of Disaster Risk Reduction, 27(1), 588-595. doi: 10.1016/j.ijdrr.2017.10.004

Sutker, P. B., Corrigan, S. A., Sundgaard-Riise, K., Uddo, M., \& Allain, A. N. (2002). Exposure to war trauma, war-related PTSD, and psychological impact of subsequent hurricane. Journal of Psychopathology and 
Behavioral Assessment, 24(1), 25-37. doi: 10.1023/A:1014049123935

Taylor, R. (1990). Interpretation of the correlation coefficient: A basic review. Journal of Diagnostic Medical Sonography, 6, 35-39. doi: 10.1177/875647939000600106

Ticehurst, S., Webster, R., Carr, V., \& Lewin, T. (1996). The psychosocial impact of an earthquake on the elderly. International Journal of Geriatric Psychiatry, 11(11), 943951. doi: 10.1002/(SICI)1099-1166(199611) 11:11<943::AID-GPS412>3.0.CO;2-B

Timmerman, M. E., \& Lorenzo-Seva, U. (2011). Dimensionality assessment of ordered polytomous items with parallel analysis. Psychological Methods, 16(2), 209-220. doi: 10.1037/a0023353

Viswanath, B., Maroky, A., Math, S., John, J. P., Benegal, V., Hamza, A., \& Chaturvedi, S. K. (2012). Psychological impact of the tsunami on elderly survivors. American Journal of Geriatric Psychiatry, 20(5), 402-407. doi: 10.1097/JGP.0b013e318246b7e9
Watson, D., \& Tellegen, A. (1985). Toward a consensual structure of mood. Psychological Bulletin, 98(2), 219-235. doi: 10.1037/00332909.98.2.219

Weems, C. F., Watts, S. E., Marsee, M. A., Taylor, L. K., Costa, N. M., Cannon, M. F., ... Pina, A. A. (2007). The psychosocial impact of Hurricane Katrina: Contextual differences in psychological symptoms, social support, and discrimination. Behaviour Research and Therapy, 45(10), 2295-2306. doi: 10.1016/j.brat.2007.04.013

Williams, B., Onsman, A., \& Brown, T. (2010). Exploratory factor analysis: A five-step guide for novices. Australasian Journal of Paramedicine, $\quad 8(3), \quad 1-13 . \quad$ doi: 10.33151/ajp.8.3.93

Manuscrito recibido: 30-04-2019 Manuscrito aceptado: 02-07-2019 


\section{ANEXOS \\ Escala de impacto psicosocial de los desastres (SPSI-D) en formato aplicable.}

Instrucciones (leer a la persona evaluada):

A continuación, tendrá la oportunidad de participar en un diagnóstico para conocer el estado en que usted se encuentra inmediatamente después de ocurrido un desastre. Para eso, le invitamos a contestar las siguientes preguntas, todas ellas referidas al (poner nombre desastre aquí). Nos gustaría que respondiera de manera sincera todas las preguntas. Tenga la plena seguridad de que la información entregada por usted será utilizada de manera completamente confidencial. Para participar es imprescindible que usted sea mayor de 18 años y que haya vivido directamente ese desastre.

Contestar todas las preguntas podría tomarle unos 10 minutos. Y, aunque las preguntas están diseñadas para ser respondidas de manera general, pueden existir algunas que eventualmente le traigan algunos recuerdos del evento. En caso de que su malestar le impida seguir contestando, podemos suspender la encuesta.

Teniendo en cuenta lo sucedido después del señale el grado de acuerdo o desacuerdo en las siguientes afirmaciones (marque con una X):

Considere: Alto desacuerdo (0) - Desacuerdo (1) - Acuerdo (2) - Alto acuerdo (3)

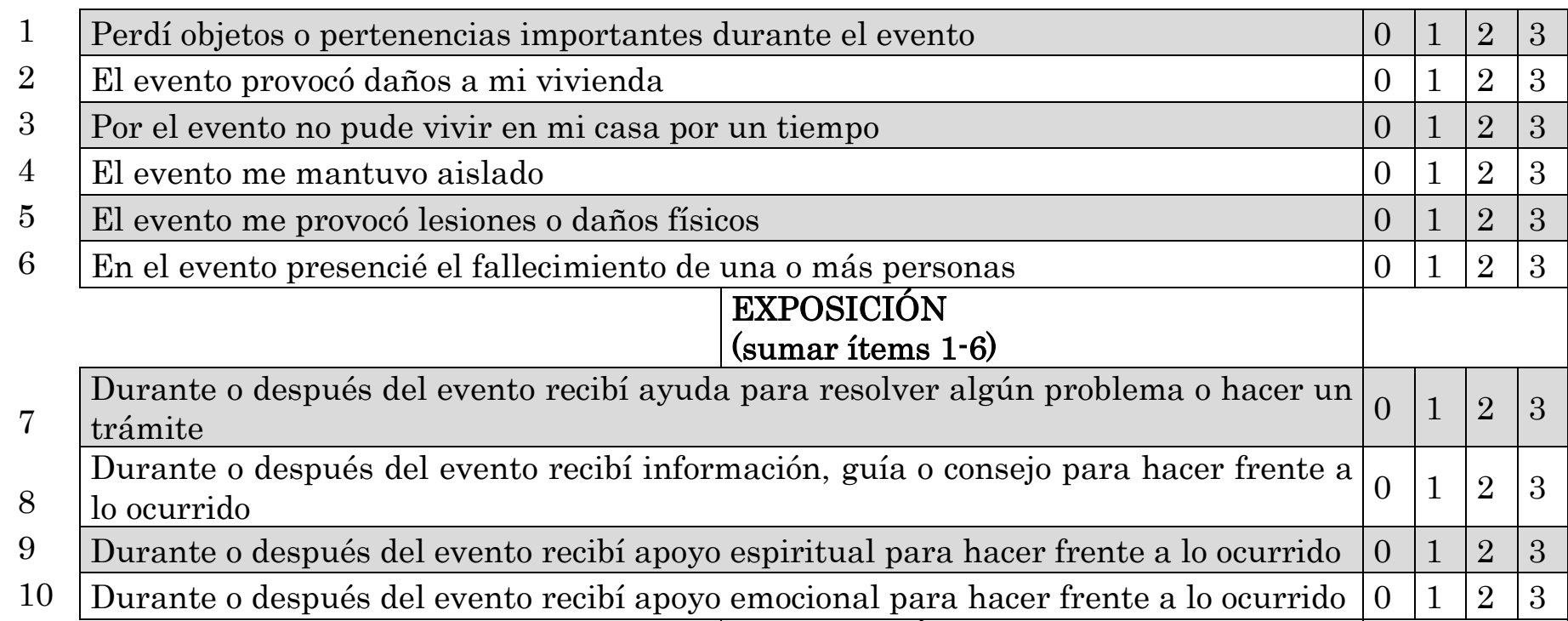

PROTECCIÓN POR APOYO SOCIAL (sumar ítems 7-10)

\begin{tabular}{|c|c|c|c|c|c|}
\hline 11 & Si la gente es buena tendrá éxito & 0 & 1 & 2 & 3 \\
\hline 12 & Las personas buenas obtienen lo que se merecen en este mundo & 0 & 1 & 2 & 3 \\
\hline 13 & La mala suerte de las personas es el resultado de los errores que cometen & 0 & 1 & 2 & 3 \\
\hline 14 & Después del evento mi fe se renovó & 0 & 1 & 2 & 3 \\
\hline
\end{tabular}


15 Busqué la lección que Dios quería entregarme

CREENCIAS DE SUERTE

(sumar ítems 11-15)

16 Tengo una buena opinión sobre mí

17 Estoy satisfecho con el tipo de persona que soy

18 Soy una persona afortunada

19 Fui capaz de hacer frente a mis problemas

20 Pensando en mi vida me he doy cuenta que he tenido suerte

21 Sentí que era capaz de tomar decisiones

22 Fui capaz de disfrutar de las actividades del día a día

\begin{tabular}{l|l|l|l|}
0 & 1 & 2 & 3 \\
\hline
\end{tabular}

AUTOCONCEPTO POSITIVO

(sumar ítems 16-22)

23 Sentí que no valía como persona

24 Me sentí un fracaso como persona

25 Perdí confianza en mí mismo

26 Perdí el gusto por las cosas o la vida

27 Tuve la sensación de estar separado de mí mismo

28 Me sentí insatisfecho o aburrido de todo

29 Tuve miedo de perder el control o volverme loco

30 Sentí culpa constantemente

31 No podía dejar de llorar aunque me lo propusiera

32 El evento afectó mis relaciones con mi familia y con amigos

33 Me fue difícil tomar decisiones

34 Me fue difícil sentir tristeza o afecto

35 Sentí que recibía un castigo

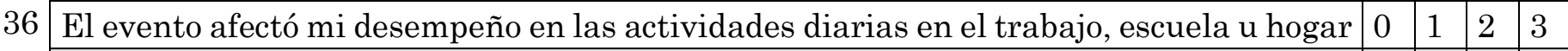

37 Me costó realizar actividades o tareas

38 El evento afectó mis actividades sociales o recreativas

39 Sentí que no podía hacer frente a mis dificultades

40 Me sentí triste o deprimido

41 Me sentí deprimido o poco feliz

42 Mi aspecto físico empeoró

Me esforcé por evitar pensar o hablar sobre lo que ocurrió o hacer cosas que me lo

43 recordaran

44 Tuve ataques de ira o estuve más irritable

45 Sentí que lo que viví no era real

46 No sentía esperanzas por el futuro 
47 Pongo más energía en mis relaciones

48 Me siento preparado para hacer mejores cosas en mi vida

49 Considero que puedo manejar mejor las cosas frente a las dificultades

50 Descubrí que soy más fuerte de lo que pensaba

51 He modificado lo que es más importante en mi vida

SALUD

(sumar ítems 47-51) 


\section{Instrucciones para el profesional que administra la escala}

Esta escala ha de ser administrada por profesionales de evaluación clínica en cualquier momento después de un desastre o emergencia.

Determine el nivel (alto o bajo) de cada dimensión del impacto psicosocial. Para eso, marque con una X la casilla que corresponde al puntaje de cada dimensión.

\begin{tabular}{|l|l|l|l|l|l|l|}
\cline { 2 - 6 } \multicolumn{1}{c|}{} & Exposición & Protección & Suerte & Autoconcepto & Disrupción & Salud \\
\hline Alta & 13 o más & 7 o más & 9 o más & 17 o más & 35 o más & 12 o más \\
\hline Baja & 0 a 12 & 0 a 6 & $0-8$ & $0-16$ & 0 a 34 & $0-11$ \\
\hline
\end{tabular}

En el círculo, marque con una $\mathrm{X}$ solo las dimensiones altas (excepto creencias y autoconcepto); achure el área resultante según tipo de impacto psicosocial (resiliente, traumático, sensible, testigo).

Finalmente, en la siguiente tabla marque según prioridad de atención.

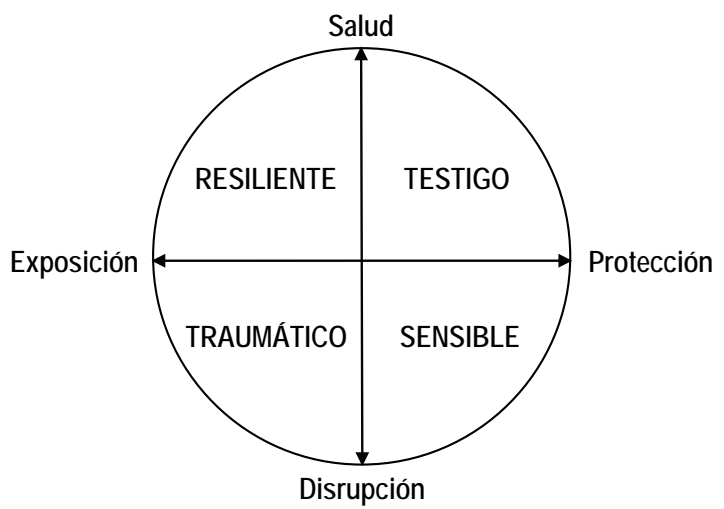

\begin{tabular}{|l|c|c|c|c|c|c|}
\cline { 2 - 7 } \multicolumn{1}{c|}{} & Exposición & Protección & Suerte & Autoconcepto & Disrupción & Salud \\
\hline 1 & Alta & Baja & Baja & Baja & Alta & Baja \\
\hline 2 & Alta & Baja & Alta & Baja & Alta & Baja \\
\hline 3 & Alta & Baja & Baja & Alta & Alta & Baja \\
\hline 4 & Alta & Baja & Alta & Alta & Alta & Baja \\
\hline 5 & Alta & Baja & Baja & Baja & Alta & Alta \\
\hline 6 & Alta & Baja & Alta & Baja & Alta & Alta \\
\hline 7 & Alta & Baja & Baja & Alta & Alta & Alta \\
\hline 8 & Alta & Baja & Alta & Alta & Alta & Alta \\
\hline 9 & Alta & Alta & Baja & Baja & Alta & Baja \\
\hline 10 & Alta & Alta & Alta & Baja & Alta & Baja \\
\hline 11 & Alta & Alta & Baja & Alta & Alta & Baja \\
\hline 12 & Alta & Alta & Alta & Alta & Alta & Baja \\
\hline
\end{tabular}

